\title{
Dark Matter Creation and Anti-Gravity Acceleration of the Expanding Universe
}

\author{
F. C. Hoh \\ Retired, Dragarbrunnsg, Uppsala, Sweden \\ Email: hoh@telia.com
}

How to cite this paper: Hoh, F.C. (2021) Dark Matter Creation and Anti-Gravity Acceleration of the Expanding Universe. Journal of Modern Physics, 12, 139-160. https://doi.org/10.4236/jmp.2021.123013

Received: December 17, 2020

Accepted: February 6, 2021

Published: February 9, 2021

Copyright (C 2021 by author(s) and Scientific Research Publishing Inc. This work is licensed under the Creative Commons Attribution International License (CC BY 4.0).

http://creativecommons.org/licenses/by/4.0/

\begin{abstract}
Dark matter is identified as negative relative energy between quarks in proton and is generated in cold hydrogen gas with pressure gradient in gravitational field. Positive relative energy PRE can be generated between quarks in protons in cold hydrogen gas in outskirts of the universe. The mechanisms for such creation of dark matter and PRE are reviewed and updated in greater detail and clearer manner. The so-generated dark matter in a galaxy can account for the galaxy's rotation curve. Star formation in this galaxy uses up the hydrogen atoms and thereby reduces its dark matter content. Dark matter created in intergalactic hydrogen gas can form filaments. In a hypothetical model of the universe, a hydrogen atom with a small amount of negative relative energy or dark matter at the outskirts of this universe can via collisions with other atoms turn into one with a small positive relative energy PRE. Once such a sign change takes place, gravitational attraction switches to anti-gravity repulsion unopposed by any pressure gradient. This leads to a "run away" hydrogen atom moving away from the mass center of the universe and provides a basic mechanism for the accelerating expansion of the universe. This theoretical expansion and the measured redshift data are both compatible with the conception of an acceleratingly expanding universe and complement each other. But they cannot verify each other directly because the present model has been constructed for purposes different from those of the measurements. But it can be shown that both approaches do support each other qualitatively under certain circumstances for small velocities. Dark matter and PRE in the present model are not foreign objects like WIMPs and dark energy-cosmological constant but can only be created in cold hydrogen gas in gravitational field. To achieve this, infrequent collisions among the hydrogen atoms must take place. Dark matter was created first and can eventually later evolve into PRE in the outskirts of the universe and in the intergalactic void. Dark matter and PRE will disappear if the hydrogen atom carrying them becomes ionized as in stars.
\end{abstract}




\section{Keywords}

Relative Energy between Quarks, Scalar Strong Interaction Hadron Theory SSI, Negative Relative Energy, Dark Matter, Positive Relative Energy PRE, Anti-Gravity Repulsion, Universe Expansion, Proton Orbit, Hubble’s Law

\section{Introduction}

\section{1. $\Lambda \mathrm{CDM}$ and General Relativity}

The present standard model of big bang cosmology $\Lambda$ CDM [1] (Lambda CDM) is based upon general relativity, a first principles' theory, augmented by additional concepts including Friedmann's scale factor and Cold Dark Matter. In the development of this subject, it is desirable to reduce the number such postulates introduced by hand and incorporate some of the aimed effects as natural outcomes of a new such theory.

Further, general relativity is a classical theory in which mass, length and hence also mass density are continuous quantities that can be 0 and $\infty$. However, the smallest mass unit contributing to baryonic matter in the universe is the proton mass, a discrete quantity $\neq 0$. In addition, the proton comprises of 3 point-like quarks which do not occupy the same spatial position and do have extension in space so that its mass density cannot be $\infty$. Therefore, general relativity breaks down at small distances and high mass densities when applied to the real universe and has to be "cut off" at suitable values from such 0 and $\infty$ where quark structure of matter enters.

\subsection{Standard Model}

In the parameter regions cut off from general relativity, an appropriate elementary particle theory is supposed to fill in. The obvious first choice is the current mainstream particle theory, the standard model SM [2]. This half century old theory is based upon a hypothetical Higgs boson. The Higgs-like boson found in 2012 [3] was assigned to it. Subsequently, however, it has not been possible to establish that this boson is the SM Higgs boson (re isospin, assigned to $W^{4} W^{-}$ bound state below). This model, including quantum-chromodynamics QCD, has turned out to be not useful; it cannot account for even the most basic meson spectra and does not describe the behavior of quarks in proton. Further, it cannot explain the existence of dark matter and dark energy.

Cosmologists have been attracted to SM by its Higgs mechanism which converts the energy created in the big bang to electron and quark masses. Such fermions obey Dirac's equation and hence are observable, as does the electron. But the so-generated quarks cannot be observed and this contradicts the Higgs hypothesis. The situation reminds me of Einstein's citation of a Bertrand Russell formulation: "Naive realism, if true, is false. Therefore, it is false". 


\subsection{SSI and PRE-Positive Relative Energy}

In its place, the scalar strong interaction hadron theory SSI has been proposed

[4] [5]. This theory has been relatively successful in treating basic aspects of meson spectra, some meson decays, kaon CP violation, quark structure of nucleon, neutron decay, baryon magnetic moment, and transition to QCD. The $125 \mathrm{GeV}$ Higgs-like boson [3] was assigned to the estimated $117 \mathrm{GeV} W^{+} W$ bound state [5] (p. 158). Of particular interest here are the interquark wave functions in proton needed in (2.1) below. In SSI, a hadron consists of quarks interacting via scalar force. The unknown relative energy between these quarks has been put to 0 . Gravitation is absent and only nonrelativistic hadrons have been treated.

Recently, it has been pointed out that such relative energy of a nucleon interacts with gravitational fields on equal footing as does the nucleon itself [6]. In cosmic situations, therefore, such relative energy needs to be restored in SSI. Negative and positive relative energies have been assigned to the inferred dark matter and dark energy, respectively [6] [7] [8].

Negative relative energy or dark matter generated in a neutron on the Schwarzschild sphere of a neutron star falling towards its center can exactly cancel the gravitational energy gained in this fall. This neutron becomes weightless and the fall is halted. This mechanism can prevent the creation of gravitational singularity [7] (Section 6 - 7). Dark matter is also generated in cold hydrogen gas in an expanding galaxy. It provides additional attractive force to keep fast moving stars from escaping this galaxy [7] (Section 8).

Positive relative energy can be generated in outskirts of the observable universe but not where dark matter is created. Conventionally, dark energy is assumed to permeate throughout the universe and has been associated with the cosmological constant, as in the $\Lambda \mathrm{CDM}$ model. Therefore, the earlier assignment of positive relative energy to dark energy in [6] [7] [8] leads to confusion and has to be retracted. Here, the following definitions

$$
\begin{aligned}
& -\omega=\text { relative energy between } u u \text { diquark and } d \text { quark in proton } \\
& \text { with mass } E_{0} \\
& \text { dark matter = negative relative energy between } u u \text { diquark and } d \\
& \text { quark in proton, }-\omega<0 \\
& \text { PRE }=\text { Positive Relative Energy between } u u \text { diquark and } d \text { quark } \\
& \quad \text { in a proton, }-\omega>0
\end{aligned}
$$

are adopted. The conventional meaning of dark energy remains unaltered. Like dark energy, PRE is also not observable but for different reasons.

\subsection{Purpose}

The above results have been obtained using simple models to illustrate the mechanisms. The purpose of this paper is to review and update these mechanisms in greater detail and clearer manner. A simple model of a galaxy and a hypothetical model of the universe have been constructed for this purpose. Further, ex- 
pansion of the universe via anti-gravity repulsion caused by PRE is treated semi-quantitatively.

In Section 2, the basic mechanism of the generation of dark matter in a galaxy model is reviewed and clarified in greater detail. The so-created dark matter phenomenologically accounts for the galaxy rotation curve and filaments. This also paves the way for the mechanism of creation of PRE in Section 3. By means of a hypothetical model of the universe, such PRE leads to anti-gravity repulsion which expands the universe in Section 4. The so-obtained nonlinear equations of motion for a test hydrogen atom participating in such expansion is solved on computer in Section 5. Relations between the so-obtained results and Hubble's law and a partial comparison to the $\Lambda \mathrm{CDM}$ model are given in Section 6 . The Appendix reproduces some earlier results for reference.

\section{Dark Matter Generation and Applications}

\subsection{Basic Mechanism for Dark Matter Creation}

The mechanism of dark matter generation [7] (Section 8), [8] (Section 4) will be explained in greater detail.

The coordinates of the diquark $u u$ at $x_{I}$ and the quark $d$ at $x_{I I}$ in a proton cannot be observed. In SSI, they have been transformed into an observable laboratory coordinate $X$ for the proton and an unobservable, "hidden" relative coordinate $x$ (A2) between $u u$ and $d$. The transformation constant $a_{m}$ can in principle be any real number. In the plane wave expansion of the proton wave function (A3), the relative energy $-\omega$ is however connected to $a_{m}$ via (A4), which insures that the proton mass and behaviour are unaffected by such a variable transformation [7] (5.1, 2), [5] (3.1.10a). The third of (A2) and (A4) then yield the ratio $R_{D}$ between the relative energy generated to the proton mass $E_{0}$,

$$
R_{D}=\frac{-\omega}{E_{0}}=-a_{m}+\frac{1}{2}=-\frac{\left|\underline{X}-\underline{x}_{I}\right|}{\left|\underline{x}_{I I}-\underline{x}_{I}\right|}+\frac{1}{2}=-\frac{X_{p}-x_{I}}{r_{a}}+\frac{1}{2}
$$

where $X_{p}$ is the proton coordinate. The time components have been left out. The distance between $u u$ and $d,\left|\underline{x}_{I I}-\underline{x}_{I}\right|$, has been approximated by its average value $r_{a}=3.23 \mathrm{fm}$ [8] (2.5) which depends upon the strong $u u-d$ potential $\Phi_{b}(r)$ (A6) and wave functions (A7) in relative space. This situation is illustrated in Figure 1 for a "test" proton at $X_{p}$ in a "test" hydrogen atom in an expanding "test" galaxy.

This test hydrogen atom is acted upon by the ambient gravitational force and eventual centrifugal force due to its motion. These forces accelerate the proton and the electron in this atom equally and move the test atom as a single entity.

Phenomenologically, an average test atom is being pushed towards the right in Figure 1 by the gas pressure gradient present due to the higher gas density and temperature in the inner part of the galaxy. This pushing force comes from collisions between this test atom and other atoms in the gas, acts on the electron of the test atom only and is a Coulomb force. In cold hydrogen gas in interstellar space, such 
collisions are elastic. The proton inside this atom is largely shielded from this force by the charge of its orbiting electron. It will however be dragged along via just this electrostatic coupling to the electron. This force tends to move the electron and proton clouds to the right in Figure 1 and increase the value of $X_{p}$ in (2.1).

On the other hand, the gravitational pull from the galaxy center also acts directly on the quarks of the proton [8] (A1-2). This pull tends to move the uu-d aggregate in the opposite direction, towards the left, as is shown on the left half of Figure 1. This leads to a decrease of $x_{I}$ in (2.1).

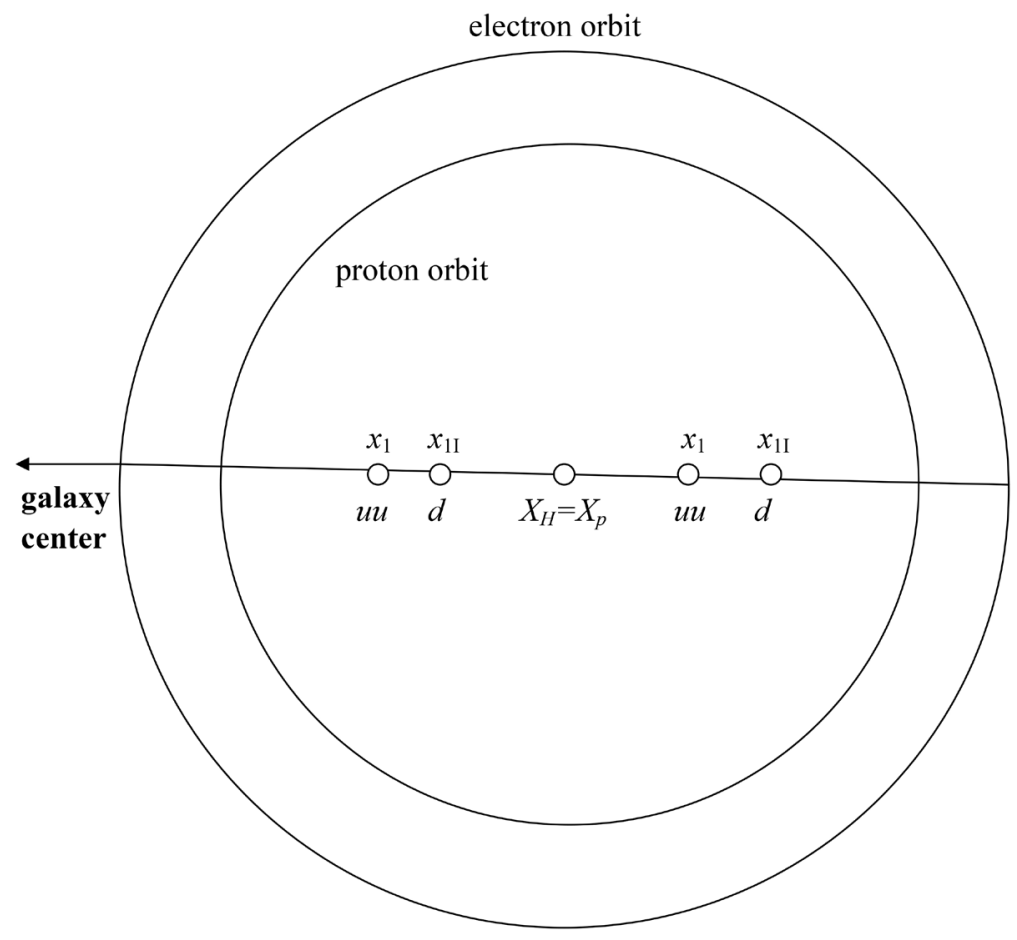

Figure 1. Schematic structure of a "test" hydrogen atom in an expanding "test" galaxy. The large electron orbit has been scaled down to fit inside this figure for illustration. Inside the proton orbit lies the center of the hydrogen atom $X_{H}$ which is also the center $X_{p}$ of the proton charge cloud having a radius of $28.8 \mathrm{fm}$ (proton Bohr radius $a_{0 p}$, inner circle) as well as the center $X_{e}$ (not shown) of the electron charge cloud having a radius of $0.53 \AA$ (Bohr radius $a_{0}$, outer circle). The aggregate of diquark $u u$ at $X_{I}$ and quark $d$ at $X_{I I}$, normally also centered at $X_{p}$, has been pulled to its left by gravitational pull from the galaxy center while the electron tends to move away from the center due to higher gas pressure closer to this center (left half of figure). These deviations from the normal, centered position generate negative relative energy between $u u$ and $d$ which behaves like dark matter and enhances the gravitational pull. Near the outskirts of the galaxy, this uu- $d$ aggregate may eventually end up on the right half of the figure after an elastic collision with another atom. Positive relative energy PRE, also invisible or dark, between $u u$ and $d$ is then generated instead of the dark matter above. This sign change of the relative energy causes this test atom to experience an anti-gravity repulsion pushing the quarks further away from the galactic center. The heavier uu diquark experiences a stronger gravitational pull from this center and therefore lies to the left of the lighter $d$ quark. The average of the $u u$ to $d$ distance $\left|\underline{x}_{I I}-\underline{x}_{l}\right|$ is $r_{a}=3.23 \mathrm{fm}$. Dark matter and PRE can be created in cold, tenuous hydrogen gas. When this gas turns into fully ionized plasma in stars, the above mechanism no longer works and no such dark constituents can be generated. 
It is this left shift of the position of the $u u-d$ aggregate relative to the position of the center of the proton and electron clouds corresponding to greater $X_{p}-x_{I}$ values in (2.1) that generates the negative relative energy or dark matter between $u u$ and $d ;-\omega<0$. If this $u u-d$ aggregate were shifted to the right, as is depicted on the right half of Figure 1, positive relative energy PRE - $\omega>0$ would be generated. This case will be treated in Section 3.

The gravitational force exerts a greater pull on the heavier diquark $u u$ than it does on the lighter quark $d$ so that uu lies to the left of $d$ and is closer to the galactic center in Figure 1. In SSI, the mass of these 3 quarks is about $50 \%$ greater than the nucleon mass [5] (Table 5.2); the difference is taken up by the strong, interquark potential $\Phi_{b}(\mathrm{~A} 6)$.

\subsection{Upper Limits of Dark Matter Generation}

The above dark matter generation mechanism is phenomenological. A formal treatment would require an integration of SSI and gravitation. This out of the scope of this paper. The mechanism in Section 2.1 holds for a single test hydrogen atom for some time before this test atom experiences a new collision and ends up in a new state in which the dark matter generated in Section 2.1 is altered or lost. Therefore, the gas containing this test hydrogen atom needs to be cold and tenuous so that collisions, necessary for such generation, are infrequent.

Under such circumstances, the direction of the pressure gradient in Section 2.1 will cause an average test proton to generate dark matter that, together with other similar hydrogen atoms, can lead to observable phenomena such as the galaxy rotation curve.

The amount of dark matter so-generated depends upon the value of the transformation constant $a_{m}$ which can so far assume any real value. The interquark separation $r_{a}$ in (2.1) depends upon the interquark strong potential (A6) in the relative space, decoupled from the laboratory space $X$, and is hence largely unaffected by an atomic collision. Thus, large $a_{m}$ implies large $X_{p}-x_{I}$ which in its turn depends upon the unknown collision parameters. However, $X_{p}-X_{I}$ can be constrained in this test atom's environment.

For example, consider a collision that kicks the electron to the right which in its turn drags the proton at $X_{p}$ along in Figure $1 ; X_{p}-X_{I}$ assumes some positive value and dark matter $-\omega<0$ is created according to (2.1). A harder collision leads to a greater $X_{p}-x_{I}$ and yields a larger amount of dark matter. But if the collision is too hard, the electron can be knocked off its orbit around the proton so that the test atom becomes ionized. In this case, the mechanism in Section 2.1 no longer works; the generated dark matter vanishes. In this case, $X_{p}-X_{I}$ is restricted by an unspecified nonionization limit. This restriction is practically satisfied because a temperature of $>50,000^{\circ} \mathrm{K}$ is needed for ionization which far exceeds the temperature of the cold hydrogen gas environment of the test atom.

A milder form of restriction on $X_{p}-X_{I}$ is the heuristic limit which requires that the $u u$ - $d$ aggregate belonging to a proton has to lie inside the proton cloud. How this can eventually be verified formally would require a formalism beyond 
the scope of this paper. This heuristic restriction limits $X_{p}-x_{I}$ in Figure 1 to the proton Bohr radius $a_{0 p}=28.8 \mathrm{fm}$ so that (2.1) becomes [8] (4.2)

$$
R_{D M}=\frac{-\omega}{E_{0}}=-a_{m}+\frac{1}{2} \geq-\frac{a_{o p}}{r_{a}}+\frac{1}{2}=-8.4
$$

This magnitude is much smaller than that due to the above-mentioned nonionization limit.

The observed average over the entire visible universe is $R_{D M} \rightarrow R_{D M E X P}=-5.6$ [1] which is not directly comparable to the heuristic $R_{D M}(2.2)$ or to the unspecified nonionization $R_{D M}$. This is due to that these two $R_{D M}$ values refer to a test atom in cold, tenuous hydrogen gas. Warm, rarified hydrogen gas and hydrogen molecule gas may eventually contribute to a less degree. On the other hand, $R_{D M E X P}$ refers to all forms of ordinary matter in the observable universe including in addition ionized media, dust, stars, planets, etc in which no dark matter can be created by the mechanism of Section 2.1. Thus, $R_{D M E X P}$ is created from the hydrogen gas part of the universe only. This part has therefore to produce $\left|R_{D M}\right|>\left|R_{D M E X P}\right|$. The actual $\left|R_{D M}\right|$ may perhaps lie around 8.4 of the heuristic (2.2) but well below the much higher, unspecified nonionization value.

This situation appears to be qualitatively compatible with Milky Way data. Milky Way has 1\% - 5\% cold hydrogen gas in volume and hence also a small \% in mass. Therefore, it is expected to have a fairly small $\left|R_{D M}\right|$ value. The Milky Way dark matter density at the sun's position is $\sim 6 \times 10^{4}$ times smaller than average mass density of the universe.

The heuristic limit $\left|R_{D M}\right|$ is essentially the number of $u u$ - $d$ aggregates with size $r_{a}=3.23 \mathrm{fm}$ that can be fitted into one side of the proton cloud with radius 28.8 $\mathrm{fm}$ in Figure 1 . For every shift of the $u u$ - $d$ aggregate away from $X_{p}$ by its own size $3.23 \mathrm{fm}$, a new dark proton is created, up to 8.4 such in (2.2). The energy needed to move $X_{p} 28.8 \mathrm{fm}$, negligible on atomic scale, is very small and is estimated to be well covered in the momentum exchange of the collision.

The so-produced negative relative energy or dark matter in a proton is on equal footing with the proton mass itself [6], as will be shown in (4.2) below. It will therefore exert extra gravitational pull on fast moving stars farther away from the galactic center and prevent them from escaping the galaxy (Figure 1 caption); it can account for the galaxy rotation curve [7] (Section 8). Similarly, it can also give rise to gravitational lensing.

Such an effect is also expected to be prevalent in the early phases of the expansions of galaxies and the universe. Near the conters, the temperature and pressure and their gradients were high and the gravitational pull was strong. The former leads to greater $X_{p}$ and the latter to smaller $x_{I}$ in Figure 1. Large $X_{p}-x_{I}$ values yield greater amount of dark matter $-\omega<0$ via (2.1). No dark "energy" is created at this stage. These results are in agreement with our current conception.

\subsection{Model for Filament Formation}

Consider the following prototype scenario. A cold hydrogen gas cloud exists 
between two galaxy clusters. Consider a cylinder of this cloud between these clusters. The gas near the cylinder surface will fall towards the cylinder axis. This gas will according to the mechanism on the left side of Figure 1, where the galaxy center is replaced by the cylinder axis, produce dark matter which tends to increase the fall speed. The gas along the cylinder axis will not produce any dark matter due to symmetry but will be compressed by the falling matter and dark matter and produce a radial pressure gradient. This cylinder then shrinks into a thin cylinder or a tube consisting mostly of dark matter except for its core which now contains hydrogen gas with high density. Galaxies may be formed along this matter and dark matter tube, resembling a filament [9]. The observed ratio of dark matter/visible matter of about 5 in such filaments is compatible to the upper limit of $\left|R_{D M}\right|<8.4$ in (2.2).

\subsection{Star Production and Decrease of Dark Matter}

According to Section 2.1, dark matter can only be created in cold hydrogen gas in gravitational field. An exception can be some neutrons in neutron stars (see Section 1.3). However, stars are also being formed from the same gas. When a hydrogen atom in this gas is used to build a new star, it gets ionized and becomes part of the hot plasma in this star. The dark matter generated by this atom is lost and the so-produced free proton can, contrary to the proton in a hydrogen atom, not generate any dark matter via the mechanism of Section 2.1. Conversion of a hydrogen atom to a proton and an electron in a star implies a loss of dark matter in the gas.

Therefore, star formation in a galaxy reduces its dark matter content. The amount of dark matter that contributes to the galaxy rotation curve is diminished by star formation. This galaxy will appear to expand faster.

Similarly, if the universe runs out of hydrogen gas, all dark matter and PRE will also vanish, except possibly in neutron stars and some other exotic objects.

In helium, the simple two-body, $u u$ and $d$, problem here turns into a manybody problem involving many quarks and relative spaces. This problem has not been investigated.

\section{Mechanism of Generation of Positive Relative Energy PRE}

The mechanism of positive relative energy PRE generation described in [7] (Section 9), [8] (Section 5) will be updated in greater detail.

As the test hydrogen atom in Section 2 moves outwards and eventually reaches the outskirts of the test galaxy, where the gas pressure gradient, eventual centrifugal force and gravitational pull acting on it become very small and nearly balance off each other so that the expansion of this galaxy nearly comes to a halt. In the absence of force acting on this hydrogen atom, its $u u$ - $d$ aggregate will move back to its normal positions centered at $X_{p}$ (see Figure 1 caption) [7] (Figure 4, position $e$ ).

Although the net outward movement of this test hydrogen atom and its like 
may nearly vanish, they will still have some small random velocities corresponding at least to the cosmic microwave background average temperature in the universe $\sim 2.8^{\circ} \mathrm{K}$. Over time, this test hydrogen atom will experience a collision with another hydrogen atom that happens to be moving inward towards the galaxy center in Figure 1. The electron orbit of the test atom will then be slightly pushed to the left. This motion drags the proton at $X_{H}=X_{p}$ also to the left via Coulomb force. This atomic force is small compared to strong interaction forces in the relative space $x$. The $u u-d$ aggregate centered in Figure 1 is $\sim 50 \%$ heavier than the proton mass (see end of Section 2.1), is thus at least phenomenologically less affected by the collision and now lies to the right of $X_{p}$. This situation is equivalent to the right half in Figure 1 (see also "lag" in Section 5.1 above 1)-3) below).

In this switched configuration, from the left half of Figure 1 in Section 2 to the right half here, the positive $X_{p}-X_{I}$ values in (2.1) in Section 2 turns negative so that the negative relative energy generated in Section 2 changes its sign in (2.1) and turns into positive relative energy. This energy has been assigned to dark energy earlier [6] (Section 6), [7] (Section 9) but is here defined as PRE in (1.3). The gravitational attraction or pull on the dark matter in the test atom in Section 2 turns now into anti-gravity repulsion or push on the PRE produced, trying to push this test atom outwards, towards the right of Figure 1. Contrary to the gravitational attraction in Section 2, which is countered by gas pressure gradient, this anti-gravitational repulsion is unopposed, even supported by the very small pressure gradient present. For sufficiently large PRE created (see (4.5) below), this test hydrogen atom will "run away" [7] (Section 9) into the intergalactic void outside the test galaxy.

Applying the assumption in Section 2 that led to (2.2), (2.1) yields the heuristic upper limit of the ratio between the so-generated PRE to proton mass [8] (5.1),

$$
R_{P R E}=\frac{-\omega}{E_{0}}=-a_{m}+\frac{1}{2} \leq \frac{a_{o p}}{r_{a}}-\frac{1}{2}=8.4
$$

which has the same magnitude as that in (2.2) due to the left-right symmetry of the proton orbit in Figure 1. There is also an analogous nonionization limit corresponding to that mentioned below (2.2). This limit will similarly not be reached due to the even lower gas temperatures here.

Again, such $R_{P R E}$ limits refer to the by now cold hydrogen test atom in the outskirts of the test galaxy. Such $R_{P R E}$ values can also not be directly compared to the measured ratio of dark energy to ordinary matter averaged over the universe $R_{D E E X P}=13.6$ [1] for analogous reasons as those given for $\left|R_{D M}\right|$ given below (2.2). $R_{D E E X P}$ refers to dark energies caused by entirely different mechanisms, i.e., cosmological constant throughout the universe in the $\Lambda \mathrm{CDM}$ model.

The above developments show that dark matter and PRE are not foreign objects in SSI, like those in the $\Lambda$ CDM model, but are generated in hydrogen gas in gravitational field and can vary and transform into each other dependent upon the positions of the uu-d aggregates relative to the centers of the proton clouds.

The present scenario is compatible with a current view that dark matter appears first and dark energy later, about $6 \times 10^{9}$ years ago. 


\section{Expansion of a Model Universe}

The mechanism in Section 3 can be applied to the expansion of the universe. Anticipating greater velocities for the accelerated test hydrogen atom, a Lorentz boost is performed on the rest frame proton whereby the heuristic limit (3.1) is approximately modified to

$$
R_{P R E}=\frac{-\omega}{\gamma E_{0}} \leq \frac{a_{o p}}{\gamma r_{a}}-\frac{1}{2}, \gamma=1 / \sqrt{1-v^{2} / c^{2}}
$$

for slow protons, where $v$ is the proton velocity and $c$ the light speed.

\subsection{SSI Model of Universe}

The following SSI model of the universe shown in Figure 2 is constructed to illustrate the expansion of the outer parts of the observable universe via the anti-gravity repulsion on the generated PRE.

Note that this model is not realistic because there is no known center of the observable universe. It is employed mainly for a semi-quantitative treatment of the anti-gravity expansion mechanism.

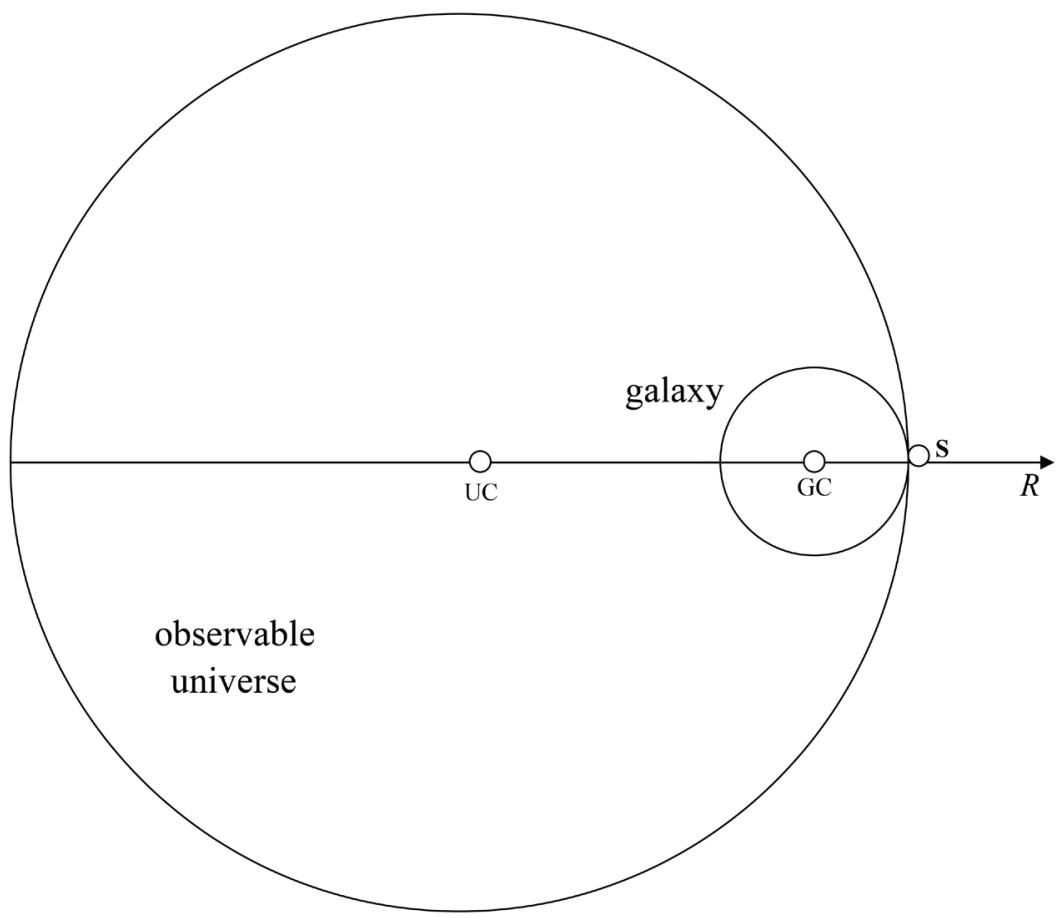

Figure 2. A hypothetical SSI model of the observable universe for illustration of the expansion of the outer parts of this universe. The large circle represents the observable universe as a sphere surrounded by vacuum. The actual boundary of this universe is not as sharp as is depicted but is somewhat diffuse. This universe is populated by galaxies with homogeneous distribution. The earth's position is unknown. The small circle represents the "test" galaxy in Figure 1 and is also a sphere lying in an outskirt of this universe. UC denotes the center of this universe and may eventually be associated with the big bang itself. $G C$ is the center of the test galaxy. $S$ denotes the point where the outer edges of both circles coincide. $R$ is the distance of the test hydrogen atom from $S$. 
From [1], the radius of the observable universe is $R_{U S}=4.4 \times 10^{26} \mathrm{~m}$. The mass of this universe is estimated from the average density $9.9 \times 10^{-30} \mathrm{~g} / \mathrm{cm}^{3}$ of the universe and its volume and is $M_{U}=3.53 \times 10^{54} \mathrm{~kg}$. The test galaxy is taken to be a copy of the Milky Way in spherical shape. The radius is $R_{G S}=100 \mathrm{kly}=9.46 \times$ $10^{20} \mathrm{~m}$ and the mass $M_{G}=2.4 \times 10^{42} \mathrm{~kg}$. This is about 3 times the mass of the Milky Way and reflects the modification of its disc form to sphere form here.

\subsection{Equation of Motion and Applications}

Let the position of the test hydrogen atom after the collision in Section 3 be $R(t)$ from $S$ in Figure 2; $t$ denotes the laboratory frame time $X^{\ominus}$ and $R(0)=0$. This test hydrogen atom will in time experience another collision. Before this happens, the proton in this atom obeys the equation of motion

$$
\begin{aligned}
& \frac{\mathrm{d}}{\mathrm{d} t} E_{0} \gamma(t) v(t)=G\left[\frac{M_{G}}{\left(R_{G S}+R(t)\right)^{2}}+\frac{M_{U}}{\left(R_{U S}+R(t)\right)^{2}}\right]\left(-\omega-E_{0} \gamma(t)\right), \\
& v(t)=\frac{\mathrm{d} R(t)}{\mathrm{d} t}
\end{aligned}
$$

where $G$ is the gravitational constant.

Inclusion of the relative energy $-\omega$ next to the proton energy $E_{0} \gamma$ in (4.2) has been demonstrated in [6] (Section 5). This can also be seen from (A3) where $-\omega x^{0}$ and $E_{0} X^{0}$ are on equal footing. Since the proton mass $E_{0}$ is known to interact with gravitational field, the associated relative energy $-\omega$ has to do so also in view that $x$ and $X$ are both linear combinations of the quark coordinates $x_{I}$ and $x_{I I}$ in (A2). Equation (4.2) is general and holds for all free hydrogen atoms.

Initially, $t$ is small, $R(t)$ can be neglected in (4.2) and $\gamma \cong 1$. Using the values given below Figure 2, (4.2) becomes

$$
\begin{gathered}
\mathrm{d} v(t) / \mathrm{d} t=-a_{c 0}\left(1+\omega / E_{0}\right), a_{c 0}=1.396 \times 10^{-9} \mathrm{~m} / \mathrm{s}^{2} \\
v(t)=v(0)-a_{c 0}\left(1+\omega / E_{0}\right) t, R(t)=v(0) t-a_{c 0}\left(1+\omega / E_{0}\right) t^{2} / 2
\end{gathered}
$$

where $v(0)$ is the initial velocity of this test hydrogen atom. About $13 \%$ of the initial acceleration $a_{c o}$ comes from the galaxy mass $M_{G}$ and $87 \%$ from the mass $M_{U}$ of the universe.

If $(4.3-4)$ were applied to the test hydrogen atom in Section $2.1, a_{c 0}$ needs to be reduced by $87 \%$. In the absence of relative energy, $-\omega=0,(4.3)$ simply describes the "free fall" of this test atom towards the galaxy center. If dark matter is generated, $-\omega<0$, and this atom will fall faster. This inward movement is on the average largely balanced off by outward movement produced by the pressure gradient in the hydrogen gas in Section 2.1.

Picking up the "run away" test hydrogen atom mentioned above (3.1), which now lies at $S$ in Figure 2. It has a small initial velocity and a PRE. The collision mentioned in the beginning of Section 3 was assumed to be strong enough to overcome the gravitational pull on the proton and produce a sufficiently large PRE $-\omega>E_{0}$. Then the right side of (4.3) becomes positive and accelerates this 
test atom outwards; the above gravitational "free fall" now turns into anti-gravity "free rise". A formal way to include such a collision in (4.2) is to put

$$
-\omega=(1+\Delta) u(t) E_{0}<\left(8.9-\frac{\gamma(t)}{2}\right) E_{0}
$$

Here, $u(t)$ is a step function; $u(t<0)=0$ and $u(t>0)=1$ representing the effect of the collision which takes place at time $t=0.1+\Delta$ is the amplitude of this step function and has to be $>1$ to overcome the gravitational pull on the test hydrogen atom; $\Delta>0$. Following the considerations on $R_{P R E}$ below (3.1), the limit (4.1) is tentatively adopted on the right of (4.5). Inserting (4.5) into (4.2) yields

$$
\begin{aligned}
& \frac{\mathrm{d}}{\mathrm{d} t} v(t)=G\left[\frac{M_{G}}{\left(R_{G S}+R(t)\right)^{2}}+\frac{M_{U}}{\left(R_{U S}+R(t)\right)^{2}}\right]\left[\frac{(1+\Delta)}{\gamma(t)}-1\right], \\
& \Delta+1<8.9-\frac{\gamma(t)}{2}
\end{aligned}
$$

For small $-\omega$ or negative relative energy $-\omega<0$, the right side of (4.2) is negative. The accompanying attraction force increases with time as $R(t)<0$ increases the magnitude of this side. Acceleration of the gravitational "fall" increases with time. On the other hand, for PRE satisfying (4.5) with $\Delta>0$, the right side of (4.6) is positive but decreases with time because $R(t)>0$ increases with time. This causes the acceleration of anti-gravity "rise" to slow down with time.

\section{Computer Solutions and Anti-Gravity Expansion of the Model Universe}

\subsection{Computer Solutions and Accelerating Expansion}

Here, the initial velocity $v(0)$ of the test atom will be taken to be the mean thermal speed of hydrogen atoms corresponding to the average temperature of the universe of $\sim 2.8^{\circ} \mathrm{K}$ mentioned in Section 3,

$$
v(0)=263 \mathrm{~m} / \mathrm{s}
$$

It is unknown how $\Delta$ in $\$ 4$ can be evaluated. It will be regarded as a free parameter here tentatively limited by the heuristic $0<\Delta<7.4$ due to (3.1) and (4.5); the associated higher nonionization limit is ignored here.

The nonlinear (4.6) with the initial conditions $R(t=0)=0, v(t=0)=v(0)$ has been solved on computers at Uppsala University. Some results are given in $\mathrm{Ta}$ ble 1 .

This table shows that the position $R(t)$ of the test atom and its velocity $v(t)$ increase with time compatible with an acceleratingly expanding universe [10]. For small $t$, these values follow the anti-gravity repulsion (4.4-5) closely. As $R(t)$ approaches first $R_{G S}$ and then $R_{U S}$ (4.6) shows that the acceleration $a_{c}$ slows down, mentioned at the end of Section 4.2. This is also reflected in Table 1. As $v(t)$ approaches the light speed, the present mainly nonrelativistic treatment of the motion of the proton in [5] breaks down and the results, marked by * in this table, are unreliable. 
Table 1. Some results from numerical integration of the equation of motion of the test hydrogen atom (4.6). $\Delta$ is defined in (4.5) and characterizes the surplus of the positive relative energy PRE generated in the test hydrogen atom after a sufficiently strong collision with another atom near $S$ in the model of Figure 2. It is a free parameter here satisfying $0<\Delta<7.4$. $R$ is the distance of this test hydrogen atom from $S$ and $v$ its speed at time $t$ after the collision. $a_{c} a_{c 0}$ is the acceleration $a_{c}=\mathrm{d} v / \mathrm{d} t$ of this test atom in (4.6) normalized to its initial value $a_{c 0}$ in (4.3) just after the collision. $H_{0}$ is the Hubble constant given in (6.1) and $H$ the Hubble parameter calculated from (6.5) below. The ${ }^{\star}$ specifies that this entry is relativistic with $\gamma>1.2$ and hence is not reliable.

\begin{tabular}{|c|c|c|c|c|c|c|c|c|}
\hline$t(\mathrm{yr})$ & 0 & $10^{4}$ & $10^{5}$ & $10^{6}$ & $10^{7}$ & $10^{8}$ & $10^{9}$ & $10^{10}$ \\
\hline \multicolumn{9}{|l|}{$\Delta=1.0$} \\
\hline$R($ ly) & 0 & $1.61 \times 10^{-2}$ & 0.821 & 74.2 & $7.32 \times 10^{3}$ & $6.82 \times 10^{5}$ & $6.43 \times 10^{7}$ & $4.97 \times 10^{9}$ \\
\hline$v(\mathrm{~m} / \mathrm{s})$ & 263.3 & 703 & $4.66 \times 10^{3}$ & $4.43 \times 10^{4}$ & $4.38 \times 10^{5}$ & $4 \times 10^{6}$ & $3.83 \times 10^{7}$ & $2.38 \times 10^{8}$ \\
\hline$a_{c} / a_{c 0}$ & 1.0 & 1.0 & 1.0 & 1.0 & 0.983 & 0.874 & 0.855 & $0.156^{*}$ \\
\hline$H / H_{0}$ & 1.0 & 2.67 & 17.7 & 167 & 1040 & 267 & 27.6 & $2.22^{*}$ \\
\hline \multicolumn{9}{|l|}{$\Delta=4.6$} \\
\hline$R($ ly) & 0 & $4.25 \times 10^{-2}$ & 3.46 & 338 & $3.33 \times 10^{4}$ & $3.05 \times 10^{6}$ & $2.85 \times 10^{8}$ & $8.85 \times 10^{9}$ \\
\hline$v(\mathrm{~m} / \mathrm{s})$ & 263.3 & 2290 & $2.05 \times 10^{4}$ & $2.03 \times 10^{5}$ & $1.98 \times 10^{6}$ & $1.8 \times 10^{7}$ & $1.64 \times 10^{8}$ & $2.95 \times 10^{8}$ \\
\hline$a_{c} / a_{c 0}$ & 1.0 & 1.0 & 1.0 & 0.999 & 0.944 & 0.87 & 0.69 & $1.5 \times 10^{-11 \star}$ \\
\hline$H / H_{0}$ & 1.0 & 8.69 & 77.9 & 740 & 2020 & 273 & 26.7 & $1.55^{*}$ \\
\hline \multicolumn{9}{|l|}{$\Delta=7.4$} \\
\hline$R($ ly) & 0 & $6.31 \times 10^{-2}$ & 5.52 & 544 & $5.33 \times 10^{4}$ & $4.87 \times 10^{6}$ & $4.35 \times 10^{8}$ & $9.32 \times 10^{9}$ \\
\hline$v(\mathrm{~m} / \mathrm{s})$ & 263.3 & 3250 & $3.28 \times 10^{4}$ & $3.06 \times 10^{5}$ & $3.16 \times 10^{6}$ & $2.88 \times 10^{7}$ & $2.38 \times 10^{8}$ & $2.98 \times 10^{8}$ \\
\hline$a_{d} a_{c 0}$ & 1.0 & 1.0 & 1.0 & 0.999 & 0.926 & 0.867 & $0.474^{*}$ & $7.5 \times 10^{-12 *}$ \\
\hline$H / H_{0}$ & 1.0 & 13.4 & 125 & 1180 & 2030 & 274 & $25.4^{*}$ & $1.48^{*}$ \\
\hline
\end{tabular}

For $v(0)=0,(4.3-5)$ show that $R, v$ and $a_{c}$ are all proportional to $\Delta$. With (4.1), (4.6) is seen to behave similarly for small $v$. These three sets of values in Table 1 then largely reduce to one multiplied by 3 different $\Delta$ values. Since $v(0)$ in (5.1) is small relative to $v(t)$ in general, results in Table 1 are not sensitive to $v(0)$ and are nearly independent of it for large $\Delta$ values. For a collision that led to $\Delta=4.6$, which corresponds to the measured average ratio $\left|R_{D M}\right|=5.6$ for dark matter below (2.2), the test hydrogen atom will reach a distance of $3 \times 10^{6} \mathrm{ly}$ with a velocity of $1.8 \times 10^{7} \mathrm{~m} / \mathrm{s} 10^{8}$ years after its collision at $S$ in Figure 2 provided that it did not collide with another atom.

As was shown beneath (4.5), $\Delta>0$. Computer runs with $\Delta<0$ leads to negative $v(t)$ and blueshift. The bulk of the random collisions will yield $\Delta<0$ and these do not contribute to expansion. But sooner or later, a subsequent collision will produce $\Delta>0$. Once this occurs, one of the colliding atoms will become of the "run away" type obeying (4.6) and starts to move outwards and leave the $S$ region in Figure 2 and cannot back off. An analogy may be to push a "test ball" onto a road with a downward slope $\propto \Delta$; it will "roll away" and cannot back up.

$\Delta$ is driven by (5.1). At the outskirts of a galaxy on an outskirt of the universe, the electron and proton clouds and the center of the $u u-d$ pair all lie at the center in Figure 1, as was mentioned in the beginning of Section 3 and in the caption 
of Figure 1. Under such circumstances, a "run away" $\Delta>0$ collision needs to move the electron and proton clouds only a few fm to the left of the uu- $d$ pair to produce $\Delta \sim 1$. Equivalently, with the electron and proton clouds remain centered in Figure 1, the right half of Figure 1 depicts this case. The heavier diquark-quark pair $u u-d$ there "lag" behind relative to the charged clouds' motion towards left.

It is this "lag" that produces PRE, positive relative energy, which is repulsed by the anti-gravity turned gravitational force from the mass inside the large circle in Figure 2. The atom containing this $u u-d$ pair, instead of "falling" towards the mass centers, will "rise" from them and moves away from them.

Analogous to the considerations above Section 2.3, the momentum exchange in such a collision is sufficiently energetic to move the charged clouds up to $a_{0 p}=$ $28.8 \mathrm{fm}$ (2.2) away from the $u u$ - $d$ pair in some of such collisions so as to produce all allowed $\Delta$ values up to 7.4 .

If the above treatment for a single test hydrogen atom is to be applied to real expansion of the universe, the following ad-hoc assumptions need made:

1) All hydrogen atoms arriving at the outskirt of the observable universe indicated by the large circle in Figure 2 will become of the "run away" test atom type near $S$ there. They obey (4.6) between collisions but are slowed down on the average by collisions with other atoms.

2) All these atoms form hydrogen gas clouds in regions outside the large circle in Figure 2 and condense to galaxies in which some stars are created. Complications with respect to different generations of stars are disregarded.

3) The protons in these stars cannot be accelerated according to (4.6) (see Section 2.4, end of Figure 1's caption). These stars are assumed to be dragged along by the moving galaxies containing them via gravitational interaction and acquire the same speeds.

\subsection{Scenarios of Expanding Model Universe}

1) With the assumptions 1)-3) above, the velocity $v(t)$ increases with time $t$ and with the distance $R(t)$ in Table 1 compatible with the observed accelerating expansion of the universe in time [10] as well as with the distance from the observer (see Hubble's law in Section 6.1 below). These results arise from the generated PRE. There are only dark matter and PRE but no dark energy in the model.

In this SSI model universe, hydrogen gas "leaks" out at its boundary (large circle in Figure 2) via PRE generated there whereby the hydrogen gas pressure also diminishes. This will maintain the gas pressure gradient there and the leak can continue. A semi-steady state scenario of the expansion of the universe at the present time is reached.

2) The scenario in 1) is derived from (4.6) and holds only for $R(t) \ll R_{U S}$ because the newly created energy in the shell with thickness $R(t)$ outside the original universe (large circle in Figure 2) has not been taken into account. In time, this shell will be filled with the "run away" hydrogen atoms with PRE and part of them will collide with each other. As was mentioned above Table 1, the bulk of 
such random collisions will yield $\Delta<0$ so that the PRE carried by the participating atoms are lost. These atoms now return on the average to their normal state of a hydrogen atom. Only a small portion of collisions will produce new $\Delta>0$ "run away" hydrogen atoms with PRE.

In this qualitative manner, the hydrogen atoms "leaked" out from the universe inside the large circle in Figure 2 are largely recovered in the shell. The universe with radius $R_{U S}$ in Figure 2 has now expanded into one with larger radius $R_{U S}+$ $R(t)$. This process is repeated as this model universe expands. As was mentioned at the end of Section 4.2, this anti-gravity accelerating expansion itself slows down with increasing $t$ and $R(t)$ according to (4.6).

3) Another scenario concerns the assumed form of (4.5). The collision of the test hydrogen atom with another atom mentioned above (3.1) causes the relative energy $-\omega$ to change its sign. This implies that $-\omega$, a constant in the relative space $x$, can depend upon the laboratory time coordinate $X^{\oplus}=t$. There is no conflict here as such gravitationally induced time dependence is negligibly weak relative to those normally associated with strong inter-quark forces. Such a time dependence was included in (4.5) in form of the step function $u(t)$ with a constant amplitude $1+\Delta$. This may be regarded to be a first order $t$ dependence. To second order, a linear dependence in form of $\beta t$, where $\beta$ is another unknown constant, may be added to the above step function and modify (4.5) to

$$
-\omega=(1+\Delta) u(t) E_{0}+\beta t
$$

Inserting this into (4.2) renders the acceleration in (4.6) to increase with time instead and enhances the rate of acceleration. The heuristic limit in (4.5 - 6) may then need be modified. Furthermore, as the expansion velocities approach the light speed, relativistic effects become important so that (4.2) as well as the treatment in the Appendix no longer holds.

\subsection{Application to Dark Energy in Intergalactic Void}

The mechanism of Section 5.2 1) for the expansion at the outskirts of the model universe can be taken over to apply to the outer edges of a galaxy, as was indicated above (3.1). The hypothetical center UC of the universe in Figure 2 is replaced by the actual galaxy center. The analogously generated PRE in the gas near the outer boundary of this galaxy will "leak" into the space outside it. Such PRE loaded hydrogen gas can play the role of the "dark energy" observed in the intergalactic void.

\section{Comparison of SSI to Hubble and $\Lambda$ CDM Scenarios}

\subsection{Estimate of Hubble's Parameter}

Evidence of the expansion of the universe comes from Hubble's law

$$
v_{H}(t)=H(t) D(t), H(0)=H_{0}=2.28 \times 10^{-18} / \mathrm{s}
$$

where $v_{H}$ denotes redshift velocities of stars in distant galaxies measured from 
the earth, $D$ the earth to stars distances, $H(t)$ the Hubble parameter, and $H_{0}$ the measured Hubble constant. Differentiation of (6.1) yields

$$
\begin{aligned}
& \frac{\mathrm{d} v_{H}(t)}{\mathrm{d} t}=H(t) v_{H}(t)+\frac{\mathrm{d} H(t)}{\mathrm{d} t} D(t), v_{H}(t)=\frac{\mathrm{d} D(t)}{\mathrm{d} t} \\
& \frac{\mathrm{d} H(t)}{\mathrm{d} t}=-H^{2}(t)+H(t) \frac{1}{v_{H}(t)} \frac{\mathrm{d} v_{H}(t)}{\mathrm{d} t}
\end{aligned}
$$

Let the earth be on a radius in Figure 2, closer to the boundary of the universe (large circle) than to its center UC. There is no loss of generality to choose this radius to be that ending at $S$ in Figure 2. Let the distance from the earth to $S$ be $R_{E S}(t)$ and the light emitting stars be located near $R(t)$, the position of the test hydrogen atom governed by (4.6). Then,

$$
D(t)=R(t)+R_{E S}(t)
$$

The Hubble constant $H_{0}$ in (6.1) cannot be derived from the present model because $R_{E S}(t)$ is unknown. Now $R_{E S}(t)$ lies inside the observable universe and changes with $t$ slowly compared to $R(t)$ for a "run away" test atom accelerated via PRE near $S$ in Figure 2. With the assumptions 1)-3) given above Section 5.2, differentiation of (6.3) together with the second of (6.2) and (4.2) leads approximately to

$$
v(t)=v_{H}(t)
$$

where the unknown $R_{E S}(t)$ drops out upon differentiation. Equations (6.4), (4.6) and the last of (6.2) leads to

$$
\frac{\mathrm{d} H(t)}{\mathrm{d} t}=-H^{2}(t)+H(t) \frac{G}{v(t)}\left[\frac{M_{G}}{\left(R_{G S}+R(t)\right)^{2}}+\frac{M_{U}}{\left(R_{U S}+R(t)\right)^{2}}\right]\left[\frac{1+\Delta}{\gamma(t)}-1\right]
$$

For small $t$, (4.6) can be replaced by (4.3 - 5) and (6.5) becomes

$$
\frac{\mathrm{d} H(t)}{\mathrm{d} t}=-H^{2}(t)+H(t) \frac{\Delta a_{c 0}}{v(0)+a_{c 0} \Delta t}
$$

For small $\Delta$, the last term can be dropped and the solution is

$$
H(t)=\frac{H_{0}}{1+H_{0} t}
$$

which reduces to the second of (6.1) at $t=0$. This deceleration is extremely weak. A $1 \%$ reduction of $H(t)$ will take $1.4 \times 10^{8}$ years.

A linearized approximation, valid for small $t$, is achieved via $H^{2}(t) \rightarrow H_{0} H(t)$ in (6.6) which now yields

$$
H(t)=H_{0}\left(1+\frac{\Delta a_{c 0} t}{v(0)}\right) \exp \left(-\frac{H_{0}}{\Delta} t\right)
$$

For $\Delta$ values in Table $1, H_{0} / \Delta \ll \Delta a_{c 0} / v(0)$ and the exponential factor in (6.8) can be dropped. This is equivalent to putting the small decelerating term $-H^{2}(t)$ in (6.6) to 0. Equation (6.8) then becomes the same as the first of (4.4) using (4.5) if $H(t)$ and $H_{0}$ were replaced by $v(t)$ and $v(0)$, respectively. Thus, the 
Hubble parameter $H(t)$ increases with time at the same rate as does the accelerating expansion $v(t)$ in (4.4) for small $t$. As was mentioned above Table 1 , this acceleration is qualitatively compatible with data [10].

This increase is due to that the last term in (6.6) arising from PRE acceleration is positive and large compared to the decelerating $-H^{2}(t)$. This last term becomes large as $H(t)$ become greater at larger times; (6.8) then no longer holds and the nonlinear (6.5) has to be solved on computer.

The computer results in Table 1 show that $H(t)$ increases initially as in (6.8). At some intermediate time, the decelerating $-H^{2}(t)$ term becomes large enough to cancel out the last, accelerating term in (6.5) so that $H(t)$ reaches very large maxima there and then starts to decrease to smaller values at larger times, of the order of magnitude of $10^{8-9}$ years. At such large times, the test atom may have collided with other atoms so that the PRE gained via (6.5) was lost and the results in Table 1 no longer hold.

These results are based upon the identification (6.4) which in its turn depends upon the gross, ad-hoc assumptions 1)-3) above Section 5.2. Therefore, the $H(t)$ predictions in Table 1 can only be taken to be qualitative estimates that indicate the mechanism involved.

\subsection{Relations to Hubble's Law}

The Hubble law (6.1) is empirical and valid in the part of the observable universe containing the earth and the galaxies visible from it. This scenario is more realistic in this respect but does not include dark matter. This law cannot be derived from any first principles' theory without introducing additional concepts. The expansion mechanism is not clear; the mainstream candidate is the unidentified dark energy.

On the other hand, in the present SSI model in Figure 2, there is a center of the universe but no specified position of the earth. This model however includes dark matter and can be connected to the first principles' theory SSI [5] partially verified by hadron data. The accelerating expansion mechanism is delineated. But the expansions predicted in this model cannot be connected to any observable data; the positions and velocities of atoms in neutral hydrogen gas cannot be measured as such.

Results from both of these approaches are compatible with the general conception of an acceleratingly expanding universe and complement each other. In the Hubble case, measurements can be made but no theory exists to account for them. In the SSI model, theory exists to explain the accelerating expansion but no measurement can be made to verify it. The theoretical results can thus not confirm data directly because they refer to different environments.

Nevertheless, with the aid of the assumptions 1)-3) above Section 5.2, the connection (6.4) could be set in a heuristic manner. This connection leads to qualitative compatability between data and present theory under such circumstances for small velocities. 
Table 2. Comparison of the $\Lambda$ CDM model of the universe with the present SSI model.

\begin{tabular}{|c|c|c|}
\hline Model & $\Lambda \mathrm{CDM}$ & SSI \\
\hline Foundation & $\begin{array}{l}\text { General relativity and } \\
\text { additional basic postulates }\end{array}$ & $\begin{array}{l}\text { First principles' theory supported } \\
\text { by hadron data. No additional } \\
\text { basic postulate }\end{array}$ \\
\hline Dark Matter & $\begin{array}{l}\text { Assumed CDM, cold dark matter, } \\
\text { WIMPs not found }\end{array}$ & $\begin{array}{l}\text { As negative relative energy generated } \\
\text { among quarks in inhomogeneous, cold } \\
\text { hydrogen gas in gravitational field }\end{array}$ \\
\hline Dark Energy & $\begin{array}{l}\text { Difficulty with the cosmological } \\
\text { constant problem }\end{array}$ & $\begin{array}{l}\text { No dark energy. Only positive relative } \\
\text { energy generated in hydrogen gas in } \\
\text { outskirts of observable universe } \\
\text { yielding anti-gravity expansion }\end{array}$ \\
\hline Distribution & $\begin{array}{l}\text { Universe has no center, no boundary, } \\
\text { supported by Hubble's law }\end{array}$ & $\begin{array}{l}\text { Universe has an unobserved center } \\
\text { and is bounded by surrounding } \\
\text { vacuum. into which it expands }\end{array}$ \\
\hline
\end{tabular}

Another approximately mutually compatible scenario may be that evolution according to the SSI model took place long, perhaps $10^{9}-10^{10}$ years, ago. Evolution of such a universe may eventually have ended up in the present state of the observable universe in which Hubble's law (6.1) can be established.

\section{3. $\Lambda$ CDM vs SSI Model}

As was mentioned in the caption of Figure 2, the present SSI scenario was originally constructed for illustration of the mechanisms for the generation of dark matter and the anti-gravity expansion of the universe via PRE and was not aimed at a model for the universe per se. It turned out however that this scenario covers several areas also considered in the current mainstream $\Lambda \mathrm{CDM}$ model. Therefore, a comparison of these two models in these areas listed in Table 2 may illuminate the pros and cons for both.

\section{Summary}

Negative relative energy between the diquark $u u$ and the quark $d$ in a proton plays the role of dark matter. Positive relative energy PRE between these quarks can lead to anti-gravity expansion of the universe.

Inside the universe, the amount of negative relative energies or dark matter produced depends upon the gravitational force acting on the quarks in hydrogen gas opposed by the pressure gradients acting on the electrons in this gas. In a galaxy, such amount can prevent fast moving stars from escaping the galaxy and cause gravitational lensing. Star formation in this gas uses up the hydrogen atoms that create such dark matter and hence reduces the dark matter content in this galaxy and increases the galaxy's apparent expansion rate. In intergalactic space, such dark matter can contribute to formation of filaments.

To account for the expansion of the universe, a hypothetical model of the un- 
iverse is proposed. Near the outskirts of the observable universe, both the gas pressure gradient and gravitational force become very weak and the amount of dark matter generated nearly vanishes. Random collisions between hydrogen atoms in this region can flip such a small amount of negative relative energy or dark matter into a small positive relative energy or PRE in a hydrogen atom. Once such a sign change takes place, gravitational attraction switches into anti-gravity repulsion now unopposed by any pressure gradient. This leads to a "run away" hydrogen atom and provides the mechanism for an acceleratingly expanding universe.

This theoretical expansion and the measured Hubble data are both compatible with the conception of an expanding universe and complement each other. But they cannot verify each other directly because the present model has been constructed for purposes different from those of the measurements. On the other hand, the present accelerating expansion mechanism of the universe is based upon a first principles' theory while Hubble's law cannot be derived from any such theory. However, both approaches can under certain circumstances be shown to support each other qualitatively at small velocities.

Dark matter and PRE are not foreign objects like WIMPs and dark energy-cosmological constant but can only be created in cold hydrogen gas in gravitational field. To achieve this, collisions among the hydrogen atoms must take place. Dark matter was created first and can eventually later evolve into PRE in the outskirts of the universe. Dark matter and PRE will disappear if the hydrogen atom creating them becomes ionized as in stars.

\section{Conflicts of Interest}

The author declares no conflicts of interest regarding the publication of this paper.

\section{References}

[1] Wikipedia (2020) https://en.wikipedia.org/wiki/Main_Page

[2] Burgess, C. and Moore, G. (2007) The Standard Model, A Primer. Cambridge University Press, Cambridge

[3] ATLAS, CMS Collaboration (2012) Phys Lett, B716, 30.

[4] Hoh, F.C. (1993) International Journal of Theoretical Physics, 32, 1111-1133. https://doi.org/10.1007/BF00671793

[5] Hoh, F.C. (2019) Scalar Strong Interaction Hadron Theory II. Nova Science Publishers, New York.

[6] Hoh, F.C. (2019) Journal of Modern Physics, 10, 635-640. https://doi.org/10.4236/jmp.2019.106045

[7] Hoh, F.C. (2019) Journal of Modern Physics, 10, 1645-1658. https://doi.org/10.4236/jmp.2019.1014108

[8] Hoh, F.C. (2020) Journal of Modern Physics, 11, 967-975. https://doi.org/10.4236/jmp.2020.117060

[9] Riordan, M. and Schramm, D.N. (1991) Shadows of Creation: Dark Matter and the 
Structure of the Universe, W H Freeman \& Co.

[10] Peebles, P.J.E. and Ratra, B. (2003) Reviews of Modern Physics, 75, 559. https://doi.org/10.1103/RevModPhys.75.559 


\section{Appendix. Equations of Motion for Baryons and Nucleon Wave Functions}

The equations of motion for baryons in SSI are [7] (2.2), [8] (A9 - A10) or [5] (9.3.16, 19), (9.2.13b), $\check{\mathrm{S}}$

$$
\begin{aligned}
& \partial_{I}^{a \dot{b}} \partial_{I}^{g \dot{h}} \partial_{I I \dot{e} f} \chi_{\{\dot{b} \dot{h}\}}^{f}\left(x_{I}, x_{I I}\right)=-i\left(M_{b}^{3}+\Phi_{b}\left(x_{I}, x_{I I}\right)\right) \psi_{\dot{e}}^{\{a g\}}\left(x_{I}, x_{I I}\right) \\
& \partial_{I \dot{b} c} \partial_{I \dot{h} k} \partial_{I I}^{d \dot{e}} \psi_{\dot{e}}^{\{c k\}}\left(x_{I}, x_{I I}\right)=-i\left(M_{b}^{3}+\Phi_{b}\left(x_{I}, x_{I I}\right)\right)_{\{\dot{b} \dot{h}\}}^{d}\left(x_{I}, x_{I I}\right) \\
& M_{b}=\left(2 m_{A}+m_{B}\right) / 2 \\
& \overline{\lceil\rceil}_{I} \overline{\rceil}_{I} \overline{\lceil\rceil}_{I I} \Phi_{b}\left(x_{I}, x_{I I}\right)=\frac{1}{4} g_{s}^{6}\left\{\chi_{\{b \dot{b}\}}^{f}\left(x_{I}, x_{I I}\right) \psi_{f}^{\{\dot{b i h}\}}\left(x_{I}, x_{I I}\right)+c . c .\right\}
\end{aligned}
$$

where the spinor indices run from 1 to $2, x_{I}$ is the coordinate of the diquark, $x_{I I}$ that of the quark, $\partial_{I}=\partial / \partial x_{I}, \partial_{I I}=\partial / \partial x_{I I}$, the $m$ 's quark masses [5] (Table 5.2), $\chi$ and $\psi$ the ground state baryon wave functions, $\Phi_{b}$ the interquark potential dependent only upon the interquark distance $\left|\underline{x}_{I I}-\underline{x}_{l}\right|$ for free baryons [5] (Sec. 10.1), and $g_{s}^{2}$ the strong quark-quark coupling constant. Introduce the observable laboratory frame $X$ and the "hidden", unobservable relative coordinates $x$ and separate $\chi$ and $\psi$ according to [8] (2.1 - 3) or [5] (3.1.3a), (3.1.10a) (10.1.1),

$$
\begin{gathered}
x^{\mu}=x_{I I}^{\mu}-x_{I}^{\mu}, X^{\mu}=\left(1-a_{m}\right) x_{I}^{\mu}+a_{m} x_{I I}^{\mu}, a_{m}=\left(X^{\mu}-x_{I}^{\mu}\right) /\left(x_{I I}^{\mu}-x_{I}^{\mu}\right) \\
\chi_{\{\dot{a} \dot{c}\}}^{e}\left(x_{I}, x_{I I}\right)=\chi_{\{\dot{a} \dot{c}\}}^{e}(\underline{x}) \exp \left(i \omega x^{0}\right) \times \exp \left(-i K_{\mu} X^{\mu}\right) \\
\psi_{\dot{e}}^{\{a c\}}\left(x_{I}, x_{I I}\right)=\psi_{\dot{e}}^{\{a c\}}(\underline{x}) \exp \left(i \omega x^{0}\right) \times \exp \left(-i K_{\mu} X^{\mu}\right), \\
K_{\mu}=\left(E_{K},-\underline{K}\right)
\end{gathered}
$$

Here, $E_{K}$ is the baryon energy, $\underline{K}$ its momentum and $\omega$ the relative energy between the diquark and the quark, and $a_{m}$ a real constant. The baryon wave functions $\chi$ and $\psi$ have 6 components each comprising of a spin 1/2 doublet part $\chi_{0 \dot{a}}, \quad \psi_{0}^{a}[5]$ (9.2.2) and a spin 3/2 quartet part $\chi_{ \pm 3 / 2}, \chi_{ \pm 1 / 2}, \psi^{ \pm 3 / 2}, \psi^{ \pm 1 / 2}[5]$ (9.2.8).

Consider the rest frame $\underline{K}=0$ doublet baryons and put [5] (3.1.10a), (Sec. 10.1) or [8] (2.4)

$$
a_{m}=1 / 2+\omega / E_{0}
$$

Substituting (A2-A4) into (A1) and put $\underline{K}=0$, (A1) can be decomposed into a quartet part for the spin $3 / 2$ baryons [5] (10.5.1) and a doublet part for the spin $1 / 2$ baryons [5] (10.2.1a),

$$
\begin{aligned}
& \left(i \delta^{a \dot{b}} E_{0} / 2+\underline{\sigma}^{a \dot{b}} \underline{\partial}\right)\left(E_{0}^{2} / 4+\Delta\right) \chi_{0 \dot{b}}(\underline{x})=i\left(M_{b}^{3}+\Phi_{b}(\underline{x})\right) \psi_{0}^{a}(\underline{x}) \\
& \left(i \delta_{\dot{b} c} E_{0} / 2-\underline{\sigma}_{b c} \underline{\partial}\right)\left(E_{0}^{2} / 4+\Delta\right) \psi_{0}^{c}(\underline{x})=i\left(M_{b}^{3}+\Phi_{b}(\underline{x})\right) \chi_{0 \dot{b}}(\underline{x}), \\
& \Delta=\partial^{2} / \partial \underline{x}^{2}
\end{aligned}
$$

For the plane wave solution in (A3), the normalized amplitude of the wave functions in (A3) with $\underline{K}=0$ vanishes so that the right side of (A1d) also drops out to yield [7] (4.2) or [5] (10.2.2a), 


$$
\Phi_{b}(\underline{x})=\frac{d_{b}}{r}+d_{b 0}+d_{b 1} r+d_{b 2} r^{2}+d_{b 4} r^{4}, \quad r=|\underline{x}|
$$

where the $d_{b}$ 's are constants. The ansatz [5] (10.3.8a) reads

$$
\begin{aligned}
& \psi_{0}^{1}(\underline{x})=\frac{1}{\sqrt{4 \pi}}\left(g_{0}(r)+i f_{0}(r) \cos \theta\right), \chi_{0 \mathrm{i}}(\underline{x})=\left(\psi_{0}^{1}(\underline{x})\right)^{*} \\
& \psi_{0}^{2}(\underline{x})=\frac{1}{\sqrt{4 \pi}} i f_{0}(r) \sin \theta \exp (i \varphi), \chi_{0 \dot{2}}(\underline{x})=-\psi_{0}^{2}(\underline{x})
\end{aligned}
$$

where $\theta, \phi$ are angles in the "hidden" relative space $\underline{x}$. Equations (A-6-7) have been inserted into (A5) which has been converted into a first order system [5] (10.4.5) which in its turn has been solved on a computer for the neutron, $\Sigma^{0}$ and $\Xi^{0}$ baryons. The associated $d_{b}$ values needed to obtain confinement, $g_{0}(r \rightarrow \infty) \rightarrow 0$ and $f_{0}(r \rightarrow \infty) \rightarrow 0$, as well as $g_{0}(r)$ and $f_{0}(r)$ themselves are given in [5] (Section 11.1.2). In particular [5] (Figure 11.1b), also reproduced in [7] (Figure 1), gives $g_{0}(r)$ and $f_{0}(r)$ for the neutron that led to correct prediction of its life [5] (Table 12.1).

Due to the small differences in the mass $E_{0}$ and quark masses (A1c) between the neutron and proton, the above neutron results can be taken over for proton here. 